\title{
La Equidad De Género En Los Agronegocios: Sector Comercio De Insumos Agropecuarios Y Servicios Veterinarios Del Cantón Machala
}

\author{
Eveligh Prado-Carpio \\ Cristhian Jover Castro-Armijos \\ Sara Castillo-Herrera \\ Cesar Quezada-Abad \\ Julián Coronel-Reyes \\ Héctor Arias-Chiriboga \\ Universidad Técnica de Machala, Republica del Ecuador
}

Doi: 10.19044/esj.2018.v14n8p140 URL:http://dx.doi.org/10.19044/esj.2018.v14n8p140

\begin{abstract}
One of the most important global challenges is the eradication of gender inequality, an indicator that is constantly evaluated, and for which the countries of the world have developed public policies aimed at increasing the levels of equity in the population. The present investigation was carried out in the city of Machala, and for which it was proposed as an objective to establish the participation of women and men in agribusiness in the trade sector of agricultural inputs and veterinary services and their characteristics and as hypotheses that in the agribusiness in the sector of agricultural inputs and veterinary services, there is a great gender inequity, which is sought to scale; for its realization was taken the information of the national cadastre of agribusiness registered in Agrocalidad in the sector of agricultural inputs and veterinary services, which are a total of 75 , of these, 35 were surveyed to determine the degree of equity and participation of the woman; the variables analyzed were the university academic degree of the local owners, the age and the reason to undertake. The data were analyzed using the chi-square test and using the statistical program SPSS 20. The results obtained demonstrate the low participation of women in this economic sector of the city of Machala.
\end{abstract}

Keywords: Agribusiness, Inputs, Equity, Gender, Veterinary Services

Resumen

Uno de los retos mundiales más importantes es la erradicación de la desigualdad de género, indicador que es evaluado constantemente, y para el 
cual los países del mundo han elaborado políticas públicas encaminadas a incrementar los niveles de equidad en la población. La presente investigación fue realizada en la ciudad de Machala-El Oro y para la cual se planteó como objetivo establecer la participación de la mujer y el hombre en los agronegocios del sector comercio de insumos agropecuarios y servicios veterinarios y sus características y como hipótesis, que en los agronegocios del sector comercio de insumos agropecuarios y servicios veterinarios existe una gran inequidad de género, la cual se busca dimensionar; para su realización se tomó la información del catastro nacional de los agronegocios registrados en Agrocalidad en el sector venta de insumos agropecuarios y servicios veterinarios, las cuales son un total de 75, de estas, 35 fueron encuestadas para determinar el grado de equidad y participación de la mujer; las variables analizadas fueron el grado académico universitario de los propietarios de locales, la edad y la razón de emprender. Los datos fueron analizados mediante la prueba de chi cuadrado y utilizando el programa estadístico SPSS 20. Los resultados obtenidos demuestran la escasa participación de la mujer en este sector económico de la ciudad de Machala.

Palabras-clave: Agronegocios, Insumos, Equidad, Genero, Servicios Veterinarios

\section{Introducción}

La agenda Objetivos de Desarrollo Sostenible (SDG) está conformada por 17 objetivos, los cuales constituyen la dimensión social, económica y ambiental, dando prioridad a la lucha contra la pobreza, el hambre e impulsando la igualdad de género (Bangert, Molyneux, Lindsay, Fitzpatrick, \& Engels, 2017). Para el empoderamiento de la mujer se demanda de un enfoque basado en los derechos humanos, ausente de los Objetivos de Desarrollo del Milenio (ODM) (Sen \& Mukherjee, 2014).

Tanto el sexo como el género son temas importantes en la toma de decisiones e influyen significativamente en la implementación de estrategias (Tannenbaum, Greaves, \& Graham, 2016).

Normas de género descriptivo y prescriptivo son sujeto de estudio frecuentemente por cuanto estas limitan o en algunos casos mejoran las aspiraciones familiares y profesionales (Meeussen, Veldman, \& Van Laar, 2016). En algunos casos las mujeres menos educadas podrían constituir el grupo más desfavorecido en términos de autonomía personal (Solé-auró \& Alcañiz, 2016).

En estos últimos años ha habido un incremento de mujeres que deciden emprender su negocio, sin embargo, sigue siendo un porcentaje inferior a los hombres (Zampetakis, Bakatsaki, Litos, Kafetsios, \& Moustakis, 2017), esto a pesar de existir estudios como el de (Sharma \& Sharma, 2015) en el cual se 
percibe a la equidad de género de forma positiva en relación al compromiso en el trabajo.

A pesar de existir un fuerte dominio aun por los varones en el sector empresarial, cada día se percibe más la igualdad de género con el argumento de que podría fortalecer la competitividad en diferentes áreas (Johansson \& Ringblom, 2017). A nivel empresarial se trabaja para la igualdad de género con la argumentación de que "Las inversiones hechas en mujeres pueden y deben medirse en términos de los ahorros de costos para las familias y las comunidades" (Roberts, 2015).

De acuerdo al reporte Global Entrepreneurship Monitor (2016), el índice de Actividad Emprendedora Temprana (TEA) para Ecuador fue de $31.8 \%$, es decir 2.2 veces mayor en comparación con las economías de eficiencia, de estos, el 52,01\% son de género masculino y el 47,99\% femenino (Lasio, Caicedo, Ordeñana, \& Samaniego, 2017). Si bien es cierto existe poca diferencia entre género, algunos factores limitan el emprendimiento femenino, uno de ellos las restricciones en cuanto al acceso a capital de riesgo (Lins \& Lutz, 2016), la educación, el sector económico, entre otros.

El trabajo de investigación es de gran importancia por cuanto identifica la equidad de género y su influencia en los emprendimientos del cantón Machala, en el sector comercio de insumos agropecuarios y servicios veterinarios, razón por la cual se planteó como objetivo identificar la participación de la mujer y el hombre en dicho sector y sus características. El presente estudio plantea como hipótesis que en los emprendimientos del sector comercio de insumos agropecuarios y servicios veterinarios existe una gran inequidad de género, la cual se busca dimensionar.

\section{Referentes conceptuales}

\section{Exclusión social y sociedad contemporánea}

En un sistema global dominado por la lógica del lucro, el tema de la exclusión social es de trascendental importancia, esto en vista de la suplantación del bienestar por la competitividad y productividad (Ladiana, 2017). En algunos países del mundo, en busca de estimular el crecimiento económico, han centrado esfuerzos en implementar programas que alienten al sexo femenino a participar de oficios especializados (Levasseur \& Paterson, 2016).

Existen sectores laborales en los cuales el desempeño de la mujer es relevante, contribuyendo valiosamente a la balanza comercial, tal es el caso del sector cultural en España (Martín-Ugedo, Mínguez-Vera, \& PalmaMartos, 2017), sin embargo, en muchos países del mundo existe todavía una brecha salarial de género, la cual es común y persistente (Schäfer \& Gottschall, 2015). 


\section{Emprendimiento y equidad de género}

El comercio, además de proporcionar bienes y servicios, también proporciona un intercambio de valores y principios, es por esta razón que las mujeres empresarias enfrentan constantes desafíos de género y éticos (Riding, Coscarella, Orser, \& Madill, 2008).

Un tema de principal atención en la actualidad es el valor de la iniciativa empresarial de género en el mercado global, lo cual permitiría fomentar en la población una mayor inclusión, mejorando así las normas de comercialización globales (Ratten, 2017).

En algunos lugares del mundo, como es el caso de Ghana, existe una inusual tasa de participación empresarial por parte de la mujer, lo cual se deriva de la presencia de este género en diferentes instituciones gubernamentales, las mismas que deciden emprender por factores tales como la necesidad (Langevang, Gough, Yankson, Owusu, \& Osei, 2015).

Entre las fuerzas impulsoras de la economía de las naciones se encuentra la iniciativa empresarial, la misma que, en algunos países es tomada por el sexo femenino, lo cual les permite resolver problemas como crear su propia fuente de ingresos y le da libertad para administrar su tiempo, el cual lo dividen entre la carrera y el hogar (Wasilczuk \& Zieba, 2008).

\section{Actividad económica de la ciudad de Machala}

El cantón Machala se ha caracterizado por la producción y exportación de banano, hecho que le ha hecho acreedor a la denominación de Capital Bananera del Mundo, cuenta con una población de 217.696 habitantes. La principal actividad económica de la población es el Comercio al por mayor y al por menor, es decir 10.9 mil establecimientos (SENPLADES, 2014). De acuerdo con la base estadística del Servicio de rentas Internas (S.R.I.), el impuesto a la renta causado durante el año 2017 en la ciudad de Machala fue de \$33.507.252.

Entre las actividades comerciales que destacan en la ciudad de Machala se encuentra la comercialización de productos y servicios agropecuarios; un total de 75 establecimientos o agronegocios entre empresas comercializadoras de productos agropecuarios y clínicas veterinarias funcionan en la ciudad, de acuerdo con el catastro de Agrocalidad.

\section{Materiales y Métodos}

Un dato importante a saber es la percepción e influencia de la equidad de género en los diferentes actores involucrados en las pymes emprendedoras en el sector de comercialización de insumos y servicios agropecuarios y veterinarios.

La ciudad de Machala cuenta, según el catastro 2016 de Agrocalidad, con un total de 75 agronegocios en el sector de comercialización de insumos 
y servicios agropecuarios y veterinarios, de las cuales se ha tomado un total de 35 como muestra, a los cuales se les aplicó una encuesta de tipo descriptiva durante el mes de Julio de 2017. Para el análisis descriptivo se emplearon tablas de contingencia para las variables edad y razón de emprender. El tamaño de la muestra se lo obtuvo mediante la siguiente fórmula de muestreo:

\section{Ecuación 1}

$$
\begin{aligned}
& n_{\mathrm{o}}=\frac{Z^{2} \cdot P \cdot Q}{E^{2}} \\
& n_{\mathrm{o}}=\frac{(1.64)^{2} \cdot(0.5) \cdot(0.5)}{(0.1)^{2}} \\
& \mathrm{n}_{\mathrm{o}}=67.24
\end{aligned}
$$

Ecuación 2

$$
\begin{aligned}
& n=\frac{n_{\mathrm{o}}}{1+\frac{n_{\mathrm{o}}}{N}} \\
& n=\frac{67.24}{1+\frac{67.24}{75}} \\
& n=\frac{67.24}{1.90} \\
& \mathbf{n}=35
\end{aligned}
$$

Para el análisis estadístico de la información obtenida se utilizó la prueba de chi cuadrado, la misma que tiene como finalidad comparar las posibles diferencias entre frecuencias observadas y las esperadas en la hipótesis, las cuales fueron contrastadas (Universidad de Barcelona, 2007), suponiendo como cierta la hipótesis nula $\left(\mathrm{H}^{\circ}\right)$ y tratando de definir hasta qué grado las observaciones registradas son coherentes con la misma. Para la tabulación de los datos se utilizó el programa estadístico SPSS 20.

\section{Resultados y discusión}

\begin{tabular}{|c|c|c|c|c|}
\hline & & \multicolumn{2}{|c|}{ Género } & \multirow[b]{2}{*}{ Total } \\
\hline & & Masculino & Femenino & \\
\hline \multirow{2}{*}{$\begin{array}{c}\text { Edad de } \\
\text { emprender }\end{array}$} & 18 a 35 años & $80,8 \%$ & $44,4 \%$ & $71,4 \%$ \\
\hline & 35 a 53 años & $19,2 \%$ & $55,6 \%$ & $28,6 \%$ \\
\hline \multicolumn{2}{|c|}{ Total } & $100,0 \%$ & $100,0 \%$ & $100,0 \%$ \\
\hline
\end{tabular}

Los instrumentos de investigación empleados permitieron obtener tablas y se utilizó la prueba Chi cuadrado para el establecimiento de la significancia entre las variables, de las cuales se obtuvo resultados y conclusiones, las cuales se detallan a continuación.

Pregunta 1. ¿A qué edad decidió emprender?

Tabla 1. Tabla de contingencia edad del emprendedor y género

Fuente: Encuesta a emprendedores en la ciudad de Machala

Autores: Elaboración propia 
Según los datos del Tabla N. ${ }^{\circ}$, del $100 \%$ de las encuestas realizadas podemos indicar que emprendedores masculinos con edades de 18 a 35 años, representa un $(80,80 \%)$, muy por encima de emprendedores con edades de 35 a 53 años con $(19,20 \%)$. De la misma forma el género femenino de emprendedoras con edades de 18 a 35 años, presenta un (44,44\%), por debajo de emprendedoras con edades de 35 a 53 con un $(55,60 \%)$.

Tabla 2. Pruebas de Chi-cuadrado

\begin{tabular}{|c|c|c|c|}
\hline & Valor & $\mathrm{gl}$ & Significación asintótica (bilateral) \\
\hline Chi-cuadrado de Pearson & $4,323^{\mathrm{a}}$ & 1 &, 038 \\
\hline N de casos válidos & 35 & & \\
\hline
\end{tabular}

Fuente: Encuesta a emprendedores en la ciudad de Machala Autores: Elaboración propia

En la tabla 2, podemos apreciar el valor-p es de 0.038 (Sig. asintótica bilateral) que se compara con el Alfa de significación (asumido como 5\%), es muy alto para rechazar Ho. Esta información denota que no hay indicios de una relación de dependencia entre ambas variables y, por lo tanto, se puede concluir que las variables no están relacionadas.

Pregunta 2. ¿Cuáles fueron sus razones para emprender?

Tabla 3. Tabla de contingencia razón de emprender y género

\begin{tabular}{|c|c|c|c|c|}
\hline \multicolumn{2}{|c|}{} & \multicolumn{2}{|c|}{ Género } & \multirow{2}{*}{ Total } \\
\cline { 3 - 4 } \multicolumn{2}{|c|}{} & Masculino & Femenino & $42,9 \%$ \\
\hline \multirow{2}{*}{$\begin{array}{c}\text { Razón de } \\
\text { emprender }\end{array}$} & Necesidad & $57,7 \%$ & & $57,1 \%$ \\
\cline { 2 - 5 } & Oportunidad & $42,3 \%$ & $100,0 \%$ & $100,0 \%$ \\
\hline \multicolumn{2}{|c|}{ Total } & $100,0 \%$ & $100,0 \%$ & \\
\hline
\end{tabular}

Fuente: Encuesta a emprendedores en la ciudad de Machala

Autores: Elaboración propia

En la tabla $\mathrm{N}^{\circ} 3$, de acuerdo a un total de 35 encuestas, se pudo conocer que el género masculino-necesidad de emprender es de (57,70\%), seguido por la Oportunidad de emprender que es de $(42,30 \%)$. En cambio, la oportunidad de emprender del sexo femenino es de $100 \%$.

Tabla 4. Prueba Chi-Cuadrado

\begin{tabular}{|c|c|c|c|}
\hline & Valor & gl & $\begin{array}{c}\text { Significación asintótica } \\
\text { (bilateral) }\end{array}$ \\
\hline Chi-cuadrado de Pearson & $9,087^{\mathrm{a}}$ & 1 &, 003 \\
\hline N de casos válidos & 35 & & \\
\hline
\end{tabular}

Fuente: Encuesta a emprendedores en la ciudad de Machala

Autores: Elaboración propia 
La prueba Chi Cuadrado efectuada con estos datos presenta el resultado ilustrado en la tabla N. ${ }^{\circ}$, según el cual se rechaza la hipótesis nula ( $\mathrm{p}=0.003$ menor que 0.05 ).

Se deduce que ambas variables son dependientes, con un nivel de confianza de $95 \%$.

\section{Conclusion}

El trabajo de investigación mediante la aplicación de encuestas y análisis test Chi-Cuadrado permitió conocer cuantitativamente la situación de la equidad en los agronegocios del sector comercio de insumos agropecuarios y servicios veterinarios del cantón Machala, el mismo permite motivar la realización de nuevos estudios en otros cantones de la provincia y el país, que sirvan de insumo para la política social.

El análisis de los datos obtenidos mediante encuesta permite concluir que no hay indicios de relación entre las variables edad de emprender y el género, en tanto que las variables razón de emprender y género resultan dependientes entre sí; el 100\% de personas del género femenino respondieron como aprovechar una oportunidad como la razón de emprender, en el caso del género masculino esta variable fue del $42,3 \%$.

Los resultados obtenidos son similares a los obtenidos en la parroquia El Retiro, del cantón Machala, en donde se pudo concluir una alta dependencia entre la equidad de género y los aspectos como necesidad y oportunidad para emprender, en tanto que, en relación con la edad de emprender existe dependencia entre las variables, es decir, a mayor rango de edad más equidad y viceversa (León, 2017).

\section{References:}

1. Bangert, M., Molyneux, D. H., Lindsay, S. W., Fitzpatrick, C., \& Engels, D. (2017). The cross-cutting contribution of the end of neglected tropical diseases to the sustainable development goals. Infectious Diseases of Poverty Poverty, 1-20. http://doi.org/10.1186/s40249-017-0288-0

2. Johansson, M., \& Ringblom, L. (2017). The Business Case of Gender Equality in Swedish Forestry and Mining - Restricting or Enabling Organizational Change. Gender, Work \& Organization. http://doi.org/10.1111/gwao.12187

3. Ladiana, D. (2017). Urban dystopias: Cities that exclude. International Journal of Sustainable Development and Planning, Vol. 12(4), 619627. http://doi.org/10.2495/SDP-V12-N4-619-627

4. Langevang, T., Gough, K. V, Yankson, P. W. K., Owusu, G., \& Osei, R. (2015). Bounded Entrepreneurial Vitality: The Mixed 
Embeddedness of Female Entrepreneurship. Economic Geography, Vol. 91(4), 449-473. http://doi.org/10.1111/ecge.12092

5. Lasio, V., Caicedo, G., Ordeñana, X., \& Samaniego, A. (2017). Global Entrepreneurship monitor Ecuador 2016. Quito. Retrieved from www.espae.espol.edu.ec/wpcontent/uploads/2017/06/ReporteGEM2016.pdf

6. Levasseur, K., \& Paterson, S. (2016). Jack (and Jill?) of All Trades ??? A Canadian Case Study of Equity in Apprenticeship Supports. Social Policy and Administration, Vol. 50(5), 520-539. http://doi.org/10.1111/spol.12125

7. Lins, E., \& Lutz, E. (2016). Bridging the gender funding gap: do female entrepreneurs have equal access to venture capital? International Journal of Entrepreneurship and Small Business, Vol. 27(2/3), 347. http://doi.org/10.1504/IJESB.2016.073993

8. Martín-Ugedo, J. F., Mínguez-Vera, A., \& Palma-Martos, L. (2017). Female CEOs, Returns and Risk in Spanish Publishing Firms. European Management Review, 12132. http://doi.org/10.1111/emre.12132

9. Meeussen, L., Veldman, J., \& Van Laar, C. (2016). Combining gender, work, and family identities: The cross-over and spill-over of gender norms into young adults' work and family aspirations. Frontiers in $\begin{array}{lll}\text { Psychology, } & \text { Vol. } & \text { 7(NOV), }\end{array}$ http://doi.org/10.3389/fpsyg.2016.01781

10. Ratten, V. (2017). Gender Entrepreneurship and Global Marketing. Journal of Global Marketing, Vol. 30(3), 114-121. http://doi.org/10.1080/08911762.2017.1316532

11. Riding, A., Coscarella, R., Orser, B., \& Madill, J. (2008). Women business owners in international trade: ethical issues, response strategies and moral progress. International Journal of Entrepreneurship and Small Business, Vol. 6(1), 133. http://doi.org/10.1504/IJESB.2008.017395

12. Roberts, A. (2015). The Political Economy of "Transnational Business Feminism." International Feminist Journal of Politics, Vol. 17(2), 209-231. http://doi.org/10.1080/14616742.2013.849968

13. Schäfer, A., \& Gottschall, K. (2015). From wage regulation to wage gap: How wage-setting institutions and structures shape the gender wage gap across three industries in 24 European countries and Germany. Cambridge Journal of Economics, Vol. 39(2), 467-496. http://doi.org/10.1093/cje/bev005

14. Sen, G., \& Mukherjee, A. (2014). No Empowerment without Rights, No Rights without Politics: Gender-equality, MDGs and the post-2015 Development Agenda. Journal of Human Development and 
Capabilities, Vol. $15(2-3)$,

188-202. http://doi.org/10.1080/19452829.2014.884057

15. SENPLADES. (2014). Cantón Machala. Quito. Retrieved from http://app.sni.gob.ec/sni-link/sni/Portal SNI 2014/FICHAS F/0701_MACHALA_EL ORO.pdf

16. Sharma, R. R., \& Sharma, N. P. (2015). Opening the gender diversity black box: Causality of perceived gender equity and locus of control and mediation of work engagement in employee well-being. Frontiers in Psychology, Vol. 6(OCT), 1-14. http://doi.org/10.3389/fpsyg.2015.01371

17. Solé-auró, A., \& Alcañiz, M. (2016). Educational attainment, gender and health inequalities among older adults in Catalonia (Spain). International Journal for Equity in Health, Vol. 15(126), 1-12. http://doi.org/10.1186/s12939-016-0414-9

18. Tannenbaum, C., Greaves, L., \& Graham, I. D. (2016). Why sex and gender matter in implementation research. BMC Medical Research Methodology, Vol. 16(145), 1-9. http://doi.org/10.1186/s12874-0160247-7

19. Universidad de Barcelona. (2007). Análisis estadístico. (C. S. González, Ed.) (Tercera ed). Barcelona.

20. Wasilczuk, J., \& Zieba, K. (2008). Female Entrepreneurship in Transitional Economies: The Case of Poland. Journal of Small Business and Entrepreneurship, Vol. 21(2), 153-169,252. http://doi.org/10.1080/08276331.2008.10593419

21. Zampetakis, L. A., Bakatsaki, M., Litos, C., Kafetsios, K. G., \& Moustakis, V. (2017). Gender-based differential item functioning in the application of the theory of planned behavior for the study of entrepreneurial intentions. Frontiers in Psychology, Vol. 8(MAR), 18. http://doi.org/10.3389/fpsyg.2017.00451 